\title{
The role of lung lobes in radiation pneumonitis and radiation -induced inflammation in the lung: a retrospective study
}

\author{
Matthew McCurdy • Derek P. Bergsma • Eric Hyun • \\ Thomas Kim • Enid Choi • Richard Castillo • \\ Edward Castillo • Thomas Guerrero
}

Received: 12 June 2012 / Accepted: 8 November 2012 / Published online: 4 December 2012

(C) Springer-Verlag Berlin Heidelberg 2012

\begin{abstract}
Objective We examined the relative response to radiation of the upper lung lobes (UL) vs. lower lung lobes (LL) of normal lung tissue using normalized $\left[{ }^{18} \mathrm{~F}\right]$-fluorodeoxyglucose (FDG) uptake per radiation dose received per lung voxel in patients treated with either photons or protons and tested for correlation of the radiation response with clinical pneumonitis..

Methods Seventy-five patients [photon $(n=51)$ or proton $(n=24)]$ treated for esophageal cancer from November 1 , 2003 to May 15, 2011 who received restaging FDG-positron emission tomography (PET) imaging 1-3 months after chemoradiation were selected. UL and LL were contoured using the major fissure as the boundary, with the right middle lobe being included in the right UL structure. Pneumonitis toxicity was scored using the Common Terminology Criteria for Adverse Events, version 4.0 based on the consensus of five clinicians. Results LL had a higher mean dose (15.6 vs. $10.4 \mathrm{~Gy}$, $p<0.001)$, higher mean standard uptake value (SUV) $(0.78$ vs. $0.56, p=0.001)$ and SUV in low-dose regions ( 0.80 vs. 0.66 for 10 to $20 \mathrm{~Gy}, p=0.001)$, and lower mean doseresponse ( 0.015 vs. $0.019, p=0.003)$ compared to the UL. The mean dose ratio of UL vs. LL $(p<0.001)$ and SUV in
\end{abstract}

\author{
M. McCurdy $\cdot$ E. Castillo \\ Department of Radiation Oncology, Baylor College of Medicine, \\ Houston, TX, USA \\ M. McCurdy \\ e-mail: mattrmccurdy@gmail.com \\ E. Castillo \\ e-mail: ecastillo3@mdanderson.org \\ M. McCurdy $•$ E. Hyun $\cdot$ R. Castillo $\cdot$ T. Guerrero $(\square)$ \\ Department of Radiation Oncology, The University of Texas MD \\ Anderson Cancer Center, Unit 97, 1515 Holcombe Blvd, \\ Houston 77030 TX, USA \\ e-mail: tguerrero@mdanderson.org \\ E. Hyun \\ e-mail: ehyun@mdanderson.org
}

the region of lung receiving $0-10 \mathrm{~Gy}(p=0.04)$, but not the dose-response ratio of UL vs. LL $(p=0.53)$, correlated with symptomatic pneumonitis. Upper lung lobes had a greater pulmonary metabolic radiation response than lower lung lobes. Greater dose to UL relative to LL and higher SUV in the low-dose region (10-20 Gy) on posttreatment PET correlated with symptomatic pneumonitis.

Keywords Radiation pneumonitis · Lung lobe · PET . Esophageal cancer

\section{Introduction}

Severe radiation pneumonitis (RP) is often fatal, with a mortality rate among nonsmall cell lung cancer patients who experienced severe symptoms reported to approach $50 \%$, even in the modern era of three-dimensional conformal radiation and intensity-modulated radiation therapy (IMRT) [1]. Reports on the effect of anatomic location irradiated on RP incidence have had conflicting findings. One group of studies found the

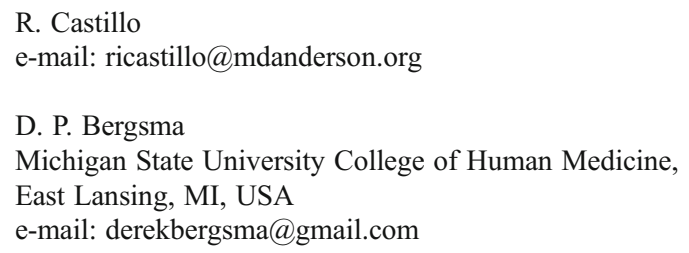

T. Kim

Warren Alpert Medical School of Brown University, Providence,

RI, USA

e-mail: kimthomas@gmail.com

\section{E. Choi}

Louisville School of Medicine, Louisville, KY, USA

e-mail: ewchoi00@gmail.com 
inclusion of superior-to-inferior tumor position as a significant factor in pneumonitis risk [2-5], while others did not [6, 7]. Rodent studies investigating the effect of superior-to-inferior irradiated location have been similarly conflicted. A greater pneumonitis response was found following irradiation of the inferior portion of the lung vs. the superior portion in some studies $[8,9]$, but not in others $[10,11]$. One group of studies hypothesizes that the correlation found in retrospective clinical and rodent studies is due to a variation in target cell density within individuals between the lobes [12]. Others have suggested any observed differences may be accounted for by interaction of the radiation response of adjacent organs, such as the heart or liver $[10,13,14]$. Hence, there remains much uncertainty as to relative radiosensitivity of the lung lobes.

Pulmonary inflammatory reactions can be visualized on $\left[{ }^{18} \mathrm{~F}\right]$-fluorodeoxyglucose positron emission tomography (FDG-PET). We found a linear relationship between pulmonary FDG uptake and radiation dose for each patient evaluated, with the slope varying over orders of magnitude between patients. The slope of this linear relation, which we refer to as the pulmonary metabolic radiation response (PMRR), was found to significantly correlate with RP clinical symptoms [15]. The PMRR $[15,16]$ provides a direct measure of the pulmonary radiation response and can directly assess differences in the lobar response.

At our institution, patients with esophageal cancer routinely undergo restaging FDG-PET/CT after chemoradiotherapy to identify patients with interval metastases [17]. In this study, we quantitatively compared the PMRR response for the upper and lower lung lobes within individuals who received thoracic radiotherapy to both the upper (UL) and lower lung lobes (LL) for esophagus cancer.

\section{Methods}

Patient radiation dose and PET data

The study population comprised patients treated in the Department of Radiation Oncology at The University of Texas MD Anderson Cancer Center for esophageal cancer between November 1, 2003 and March 15, $2011(n=75)$ who had CT treatment planning and follow-up PET/CT imaging between 25 and 75 days after completion of radiotherapy at our institution on a General Electric Discovery ST PET/CT scanner (GE Medical Systems) for disease restaging purposes. To select cases with sufficient upper and lower lobe irradiation, each case had to meet the following criteria: Volume receiving $\geq 5$ Gy must be $\geq 30 \%$, and volume receiving $\geq 40$ Gy must be $\geq 2 \%$. Patient identifiers were removed in accordance with a retrospective study protocol approved by our Institutional Review Board in compliance with the Health Insurance Portability and Accountability Act of 1996 regulations.
Each patient had undergone treatment planning CT imaging of the entire thorax and upper abdomen without contrast at 3-mm slice spacing on a Philips MX8000 IDT treatmentplanning CT scanner (Philips Medical Systems, Cleveland, $\mathrm{OH}, \mathrm{USA}$ ) or at 2.5 -mm slice spacing on a General Electric Discovery ST PET/CT scanner (GE Medical Systems, Waukesha, WI, USA) with a 70-cm bore. The radiation dose was calculated either with a free-breathing treatment planning CT (most cases) or with an average CT calculated from a fourdimensional CT (4D CT) image set [18]. The radiation dose was calculated either with a free-breathing treatment planning CT (most cases) or with an average CT calculated from a 4D CT image set $[18,19]$. Use of the average CT for attenuation correction had been introduced at MD Anderson in 2006 [20]. Approximately two thirds of the PET/CT images used in this study were attenuation corrected with mid-inspiratory breathhold obtained on the PET/CT scanner, and the remaining used an average CT obtained from a 4D CT acquisition on the PET/ CT scanner. All treatment radiotherapy plans and field arrangements were prospectively reviewed in quality assurance meetings in which consensus was obtained according to each patient's clinical circumstances.

Image registration and lung segmentation

The treatment plan for each patient was imported into a research dosimetry workstation and evaluated with the Pinnacle [3] version 7.6c or 8.0u treatment-planning system (Philips Medical Systems, Andover, MA, USA). The radiation dose distributions were all recalculated using a collapsed-cone convolution algorithm with lung heterogeneity corrections [21].

Lung parenchyma was segmented manually in Pinnacle and exported for analysis. Regions of interests (ROIs) were drawn using the major fissures to delineate the lung lobes (Fig. 1a). Left and right sided lobes were contoured separately. Since the horizontal fissure is complete in only one third of cases, the middle lobe (blue in Fig. 1a) was included in the UL ROI. The treatment plan for each patient with corresponding DICOM CT images was imported.

Lung segmentation was also applied to the CT image from the restaging PET/CT and used to aid image registration. These segmentations were manually created using a custom MATLAB (Mathworks, Inc.) program. Restaging FDG-PET/CT was imported and spatially registered to the planning CT using an affine transformation algorithm. The registered images were visually verified.

\section{Calculation of ${ }^{18}$ F-FDG uptake}

The standard uptake values (SUVs) were calculated from the PET count rate. The mean SUV within the pulmonary tissue was obtained for each case. Using the registration between the treatment-planning $\mathrm{CT}$ and the $\mathrm{CT}$ from the 
Fig. 1 A representative case. a 2D transverse, sagittal and coronal sections of planning CT with contoured lobes. Green UL; blue ML; red LL. b The normalized FDG uptake response per voxel versus radiotherapy dose (in Gy) with the linear regression result of the UL and LL. The FDG uptake response is normalized to the unirradiated ( $0-5$ Gy) lung response, allowing each case to act as its own internal control. The regression slope is the PMRR for each lobe a

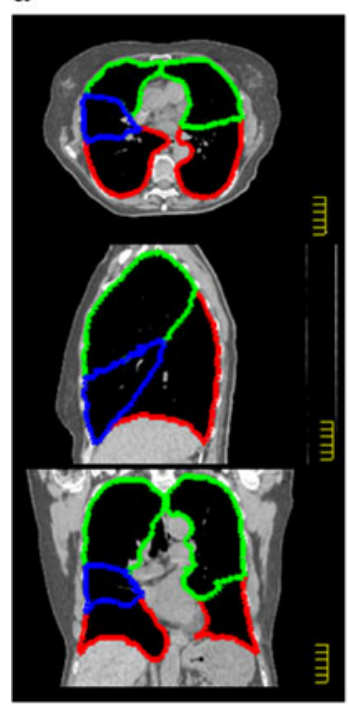

b

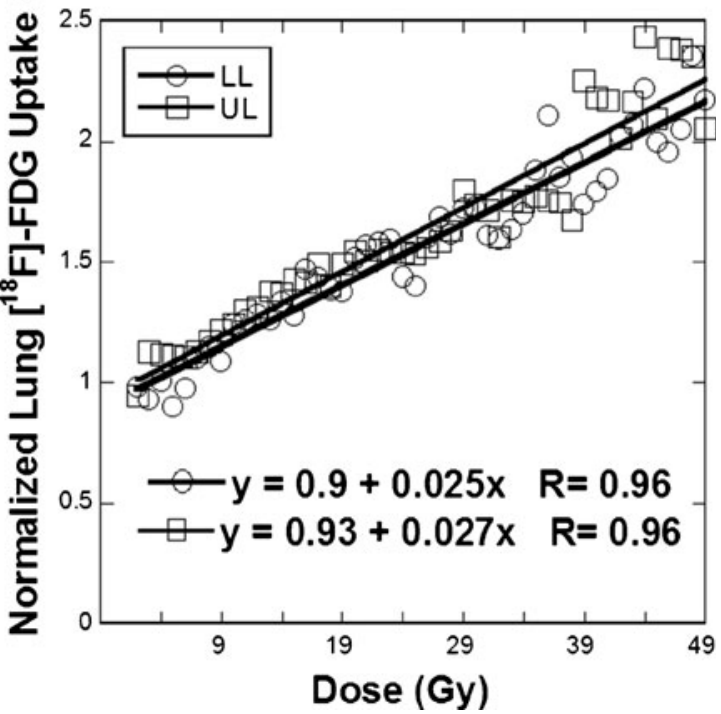

$\mathrm{PET} / \mathrm{CT}$, the mean SUV values in the lung tissue were obtained for each case over the dose ranges of $0-5,5-10$, 10-20 Gy, and subsequently in 10-Gy intervals to $50 \mathrm{~Gy}$. The median of SUV mean values and the range of the means for the 75 cases were determined.

Histograms were formed of the FDG PET count rate vs. radiation dose in 1-Gy intervals. A linear regression model was applied to the normalized $\left[{ }^{18} \mathrm{~F}\right]-\mathrm{FDG}$ uptake to obtain the PMRR for each case.

Statistical analysis

Differences in means of variables (PMRR, mean dose, and SUV) were determined using the nonparametric-related samples Wilcoxon signed rank test. A general linear model was used to test for correlation of continuous and ordinal variables with symptomatic (grade $\geq 2$ ) pneumonitis. All tests were two-sided with $p$ values of 0.05 or less considered significant. Statistical analysis was done with SPSS release 11.5.0 (SPSS, Inc., Chicago, IL, USA).

\section{Results}

Patient characteristics, dosimetric parameters, and PET parameters

Patient and clinical data are summarized in Table 1. A wide range of values for all parameters were seen depending on tumor location and treatment field design. The majority of cases were stage III. All patients received a total radiation dose of 50.4 gray or cobalt-gray equivalent and received concurrent chemotherapy. Tumor location, defined by distance from the incisors reporting during an esophagogastroduodenoscopy
Table 1 Patient and clinical characteristics

\begin{tabular}{|c|c|c|}
\hline Characteristic & Descriptor & \\
\hline \multirow[t]{2}{*}{ Age (years) } & Median & 64 \\
\hline & Range & $42-82$ \\
\hline \multirow[t]{2}{*}{ Gender $(n)$} & Male & 57 \\
\hline & Female & 18 \\
\hline \multirow[t]{5}{*}{ Stage $(n)$} & I & 0 \\
\hline & IIA & 11 \\
\hline & IIB & 4 \\
\hline & III & 45 \\
\hline & IV & 13 \\
\hline \multirow[t]{3}{*}{ Location } & Proximal & 3 \\
\hline & Middle & 29 \\
\hline & Distal/GEJ & 43 \\
\hline \multirow[t]{3}{*}{ Radiation } & 3D-CRT & 8 \\
\hline & IMRT & 43 \\
\hline & Protons & 24 \\
\hline \multirow[t]{3}{*}{ Smoking } & Never & 20 \\
\hline & Former & 52 \\
\hline & Current & 3 \\
\hline \multirow[t]{6}{*}{ Chemotherapy } & $5 \mathrm{FU}$ & 1 \\
\hline & $5 \mathrm{FU} /$ taxane & 44 \\
\hline & 5FU/oxaliplatin & 20 \\
\hline & 5FU/oxaliplatin/taxane & 8 \\
\hline & Capecitabine & 2 \\
\hline & Irenotecan/platinum & 1 \\
\hline \multirow[t]{3}{*}{ Days to PET } & Median & 40 \\
\hline & Range & $25-75$ \\
\hline & Standard deviation & 10 \\
\hline
\end{tabular}

Days to PET=days between the last day of radiotherapy and the posttreatment $\mathrm{PET} / \mathrm{CT}$ 
Table 2 Summary statistics of pulmonary metabolic radiation response (PMRR) by lobe

\begin{tabular}{lllllllll}
\hline & MLD & PMRR & SUV $_{0-10}$ & SUV $_{10-20}$ & SUV $_{20-30}$ & SUV $_{30-40}$ & SUV $_{40-50}$ & Mean SUV \\
\hline Upper lobe & 10.4 & 0.019 & 0.22 & 0.66 & 0.79 & 0.95 & 1.08 & 0.56 \\
Lower lobe & 15.6 & 0.015 & 0.25 & 0.80 & 0.95 & 0.97 & 1.13 \\
$p$ value & $<0.001^{*}$ & $0.002^{*}$ & $0.028^{*}$ & $<0.001^{*}$ & $0.002^{*}$ & 0.5 & 0.29 & $<0.001^{*}$ \\
\hline
\end{tabular}

All dose values are in gray $(\mathrm{Gy})$ or cobalt ${ }^{60}$ gray equivalent $(\mathrm{CGE})=1.1 \times \mathrm{Gy}$ and the PMRR's in $\mathrm{Gy}^{-1}$ or CGE ${ }^{-1} \cdot p$ value is for analysis of variance $M L D$ mean lung dose, SUV standard uptake value

procedure, ranged from proximal to distal, with all cases having both UL and LL irradiation dosimetrically. The mean lobe dose was calculated for the uni- and bilateral UL and LL. The mean lung dose to the total lung was 14.9 (range, 7.820.9)Gy. Mean dose to the UL was 10.4 Gy (range, 1.6-23.3) and to the LL was $15.6 \mathrm{~Gy}$ (range, 4.3-27.5) $(p<0.001)$. The median time between radiotherapy completion and the restaging FDG-PET/CT imaging session was 40 days (range, 2575 days). The mean SUV in the lung lobes was calculated for each of the 75 cases. The median value of the 75 patients of the mean SUV of the UL was 0.56 (range, 0.28-1.05) and of the LL was 0.78 (range, $0.43-1.52$ ). The median value of the 75 patients of the mean standard uptake value in each individuals lungs that received 0-5 Gy was 0.59 (range, 0.381.15), 5-10 Gy was 0.78 (range, 0.40-1.29), 10-20 Gy was 0.78 (range, $0.42-1.70$ ), and $>20$ Gy was 1.07 (range, 0.462.23). No correlation was found between the mean lung SUV value and the time between radiotherapy completion and the $\mathrm{PET} / \mathrm{CT}$ imaging session (restaging PET delay time).

\section{Clinical toxicity}

The toxicity scores were as follows: grade 0 for $14(18.6 \%)$, grade 1 for 45 (60\%), grade 2 for $9(12 \%)$, grade 3 for 5 $(6.6 \%)$, grade 4 for $0(0 \%)$, and grade 5 for $2(2.6 \%)$. The rate of clinical pneumonitis (grade $\geq 2$ ) was $21 \%$. The rate of pneumonitis was higher for proton therapy (33\%) compared to photon therapy $(15 \%)(p=0.04)$.

PMRR was obtained by regressing FDG uptake against dose with both intercept and slope in the model. A representative case is illustrated in Fig. 1. Figure 1a depicts the color-shaded UL and LL on transverse and coronal sections of the planning CT. The normalized FDG uptake response per voxel vs. radiotherapy dose (in Gy) is shown for this case along with the linear regression result of the UL and LL in (Fig. 1b). The median and range PMRR for the lower lobes was $0.015(0.0010-0.071)$ and for the upper lobes was $0.019(0.0022-0.065)$. The UL had a higher PMRR than the LL $(p=0.003)$. Figure 2 shows a scatter plot of the PMRR for the UL and LL. SUV in the region of lung receiving $0-10$ Gy was higher in the LL. Summary statistics of the Wilcoxon signed rank test are detailed in Table 2.
Analysis of variance was performed using a general linear model incorporating PMRR ratio, mean lobe dose ratio, mean SUV, SUV of lung receiving $0-10 \mathrm{~Gy}$, and SUV of lung receiving $10-20 \mathrm{~Gy}$. The PMRR ratio $(p=0.53)$ and mean SUV ( $p=0.23$ ) were not correlated with grade $\geq 2$ pneumonitis. Mean lobe dose ratio $(p<0.001)$ and SUV $10-20$ Gy ( $p=0.046)$ were correlated with grade $\geq 2$ pneumonitis. Figure 3 shows a scatter plot of the mean lobe dose ratio for asymptomatic (grade $0-1$ ) and symptomatic (grade $\geq 2$ ) pneumonitis. Data by toxicity group and analysis of variance data are summarized in Table 3.

\section{Discussion}

The primary aim of this study was to determine if there was a difference in the relative radiosensitivities of the upper and lower lung lobes in patients receiving thoracic radiation for mediastinal tumors. We found that the upper lobes had a

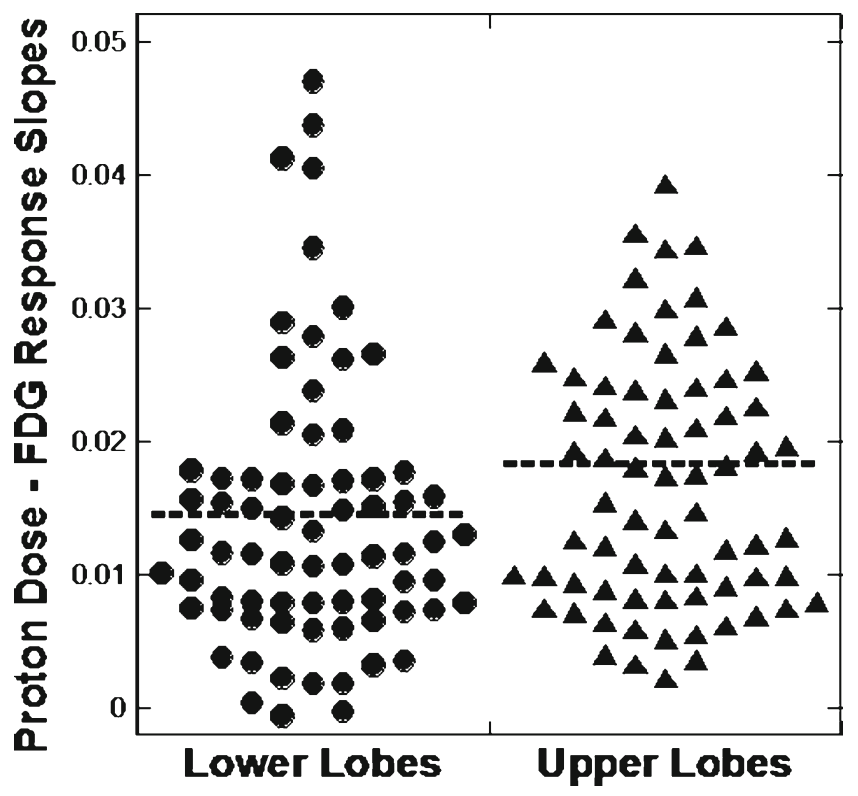

Fig. 2 Lower lobe versus upper lobe dose-response. Dot plot of the slope of the FDG uptake versus proton dose-response for 75 pairs of combined lower lobes and upper lobes. The upper lobes exhibited a significantly higher proton dose-FDG response $(p=0.003)$ when evaluated with related samples Wilcoxon signed rank test 


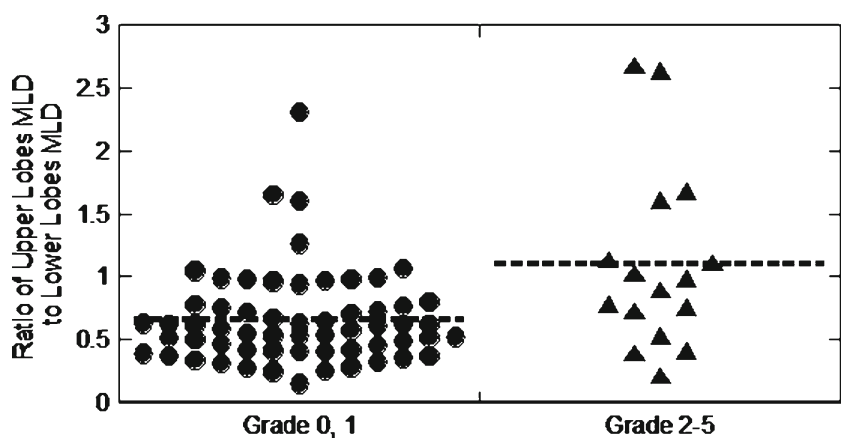

Fig. 3 Correlation of upper lobes MLD/lower lobes MLD ratio and radiation pneumonitis toxicity. Scatter plot of the ratio of the mean lung dose (MLD) of the upper lobes to the lower lobes for 75 cases. The ratio correlated with the Common Toxicity Criteria for Adverse Events version 4 radiation pneumonitis toxicity $(p<0.001)$

greater radiation dose-response than the lower lobes using the relationship of the normalized FDG uptake vs. radiation dose. This greater response on PET was not associated with a greater risk of radiation pneumonitis using a ratio of the PMRR for upper vs. lower lobes. Instead, a greater relative dose to the upper lobes and higher SUV in regions receiving low dose of radiation for the entire lung predicted clinically apparent pneumonitis. One explanation for these findings is that the acute inflammation in the areas of lung receiving 0 10 Gy represents a relatively large volume of lung that is generally $0.5 \mathrm{~cm}$ or more from the esophagus. The PMRR includes high-dose regions $>20$ Gy that are close to the esophagus. If cytokines from the esophagus diffuse into the lung and cause pneumonitis (one hypothesis), then the cytokines would have a greater impact on the PMRR than on the SUV in the region receiving 10-20 Gy because of the close proximity of the high-dose regions to the esophagus. The SUV in the 0-20 Gy range is less sensitive to anatomic changes between the planning $\mathrm{CT}$ and posttreatment PET and registration errors since there is a steep dose gradient in the lung near the esophagus tumor. Very low dose radiation $(<5$ Gy) may not produce enough response to be predictive.

The effect of anatomic location irradiated on radiation pneumonitis incidence is controversial. As an example, Hope et al. [2] developed a novel spatial and dosimetric retrospective analysis to study the effect of the location of the gross tumor volume on incidence of radiation pneumonitis in patients with lung cancer. They found that inferior tumor position had the highest univariate correlation with radiation pneumonitis among variables tested and that a model combining inferior tumor position with dose and volume information was more strongly correlated with radiation pneumonitis than any individual parameter. Bradley et al. [4] subsequently included the superior-inferior location difference in a nomogram to predict the risk of radiation pneumonitis. Two rodent studies support their clinical finding, demonstrating irradiation of the heart enhances RP symptoms [13]. Alternatively, Robnett et al. [6] retrospectively studied lung cancer patients to identify factors that may predict for severe radiation pneumonitis. Tumor location including lobe involved (upper vs. mid/lower) were not associated with severe pneumonitis risk. While most studies were carried out in lung cancer patients, the present study used esophagus cancer patients to evaluate the radiation response of relatively healthy lung. The present study defined upper and lower lobes anatomically to determine lobe sensitivity similar to Robnett et al.

One group of studies hypothesizes that the correlation of anatomic location and radiation pneumonitis found in retrospective clinical studies and rodent studies is due to a variation in target cell density within individuals between the lobes [12]. Animal studies have suggested that any observed differences may be accounted for by interaction of the radiation response of adjacent organs, such as the heart or liver [10, 13, 14]. Theuws et al. [22] found that the dose-effect relations for early changes in perfusion and ventilation were similar in shape. Both perfusion and ventilation changes showed an almost linear increase in the reduction in local function as a function of dose. They reported no regional sensitivity difference in the loss of pulmonary perfusion or ventilation radiation response. With loss of ventilation or perfusion as the endpoints, regional differences in radiosensitivity could not be demonstrated.

The rate of symptomatic pneumonitis with proton therapy was twice the rate of pneumonitis with photon therapy. This difference in pneumonitis is not accounted for by dose. The mean lung dose to the total lung for patients receiving

Table 3 Summary statistics of mean lung dose, pulmonary metabolic radiation response (PMRR) by toxicity group, and standard uptake value (SUV)

\begin{tabular}{llllllllllll}
\hline & $N$ & Mean dose & Mean dose ratio & PMRR & PMRR ratio & SUV $_{0-10}$ & SUV $_{10-20}$ & SUV $_{20-30}$ & SUV $_{30-40}$ & SUV $_{40-50}$ & Mean SUV \\
\hline Asymptomatic & 6 & 12.7 & 0.6 & 0.15 & 2.5 & 0.27 & 0.72 & 0.85 & 0.89 & 1.01 & 0.67 \\
Symptomatic & 14 & 12.0 & 1.5 & 0.18 & 2.0 & 0.47 & 0.88 & 1.12 & 1.10 & 1.32 & 0.72 \\
$p$ value & - & 0.29 & $<0.001^{*}$ & 0.13 & 0.12 & $0.04^{*}$ & 0.35 & .14 & 0.51 & 0.52 & 0.59 \\
\hline
\end{tabular}

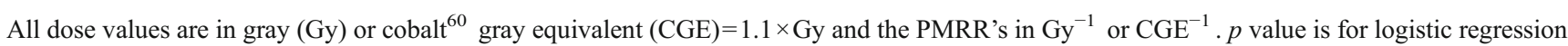
$M L D$ mean lung dose; $M L D$ ratio MLD upper lobes/MLD lower lobes; $S U V_{0-10}$ mean SUV in region of lung receiving 0-10 Gy or CGE; SUV $10-20$ $10-20$ Gy or CGE; $S U V_{20-30} 20-30$ Gy or CGE; $S U V_{30-40} 30-40$ Gy or CGE; $S U V_{40-50} 40-50$ Gy or CGE 
photon therapy was 14.5 Gy and for patients receiving proton therapy was $9.8 \mathrm{~Gy}$. The higher rate of pneumonitis with proton therapy is an area of future study.

This retrospective study has several limitations. Baseline PET studies were not incorporated since many outside PET scans were not available. Baseline PET could be used to determine if there was pre-existing inflammation of other etiology. The time from the last day of radiation treatment to restaging $\mathrm{PET} / \mathrm{CT}$ ranged from 25 to 75 days. This broad range in restaging PET may affect the mean SUV, SUV in 10 Gy dose regions, and the dose-response (PMRR). The volume effect was considered in the linear model. However, the cases where UL dose is greater than LL dose may still reflect a greater volume of total lung receiving radiation in these cases.

\section{Conclusion}

In this study, we showed that upper lung lobes have a higher FDG-PET radiation dose-response compared to lower lung lobes. The FDG-PET radiation dose-response did not predict for clinically apparent pneumonitis. A higher ratio of mean dose to the lower lung lobes and higher SUV in the region receiving $0-10$ Gy to the entire lung predicted pneumonitis.

Acknowledgments We most sincerely thank the National Institutes of Health (NIH) and the National Cancer Institute (NCI) who provided support for this project through NIH/NCI Grants R21CA141833 and T32CA119930. Additional funding for this study was provided from our institution's Physician Scientist Program.

Conflicts of interest None

\section{References}

1. Wang JY, Chen KY, Wang JT et al (2002) Outcome and prognostic factors for patients with non-small-cell lung cancer and severe radiation pneumonitis. Int J Radiat Oncol Biol Phys 54(3):735-741

2. Hope AJ, Lindsay PE, El Naqa I et al (2006) Modeling radiation pneumonitis risk with clinical, dosimetric, and spatial parameters. Int J Radiat Oncol Biol Phys 65(1):112-124

3. Seppenwoolde Y, De Jaeger K, Boersma LJ, Belderbos JS, Lebesque JV (2004) Regional differences in lung radiosensitivity after radiotherapy for non-small-cell lung cancer. Int J Radiat Oncol Biol Phys 60(3):748-758

4. Bradley JD, Hope A, El Naqa I et al (2007) A nomogram to predict radiation pneumonitis, derived from a combined analysis of RTOG 9311 and institutional data. Int J Radiat Oncol Biol Phys 69(4):985-92

5. Graham MV, Purdy JA, Emami B et al (1999) Clinical dosevolume histogram analysis for pneumonitis after $3 \mathrm{D}$ treatment for non-small cell lung cancer (NSCLC). Int J Radiat Oncol Biol Phys 45(2):323-329

6. Robnett TJ, Machtay M, Vines EF, McKenna MG, Algazy KM, McKenna WG (2000) Factors predicting severe radiation pneumonitis in patients receiving definitive chemoradiation for lung cancer. Int J Radiat Oncol Biol Phys 48(1):89-94

7. Hernando ML, Marks LB, Bentel GC et al (2001) Radiationinduced pulmonary toxicity: a dose-volume histogram analysis in 201 patients with lung cancer. Int J Radiat Oncol Biol Phys 51 (3):650-659

8. Khan MA, Van Dyk J, Yeung IW, Hill RP (2003) Partial volume rat lung irradiation; assessment of early DNA damage in different lung regions and effect of radical scavengers. Radiother Oncol 66 (1):95-102

9. Liao ZX, Travis EL, Tucker SL (1995) Damage and morbidity from pneumonitis after irradiation of partial volumes of mouse lung. Int J Radiat Oncol Biol Phys 32(5):1359-1370

10. Wiegman EM, Meertens H, Konings AW, Kampinga HH, Coppes RP (2003) Loco-regional differences in pulmonary function and density after partial rat lung irradiation. Radiother Oncol 69(1):11-19

11. Plathow C, Li M, Gong P et al (2004) Computed tomography monitoring of radiation-induced lung fibrosis in mice. Invest Radiol 39(10):600-609

12. Travis EL, Liao ZX, Tucker SL (1997) Spatial heterogeneity of the volume effect for radiation pneumonitis in mouse lung. Int J Radiat Oncol Biol Phys 38(5):1045-1054

13. Novakova-Jiresova A, van Luijk P, van Goor H, Kampinga HH, Coppes RP (2005) Pulmonary radiation injury: identification of risk factors associated with regional hypersensitivity. Cancer Res 65(9):3568-3576

14. van Luijk P, Novakova-Jiresova A, Faber H et al (2005) Radiation damage to the heart enhances early radiation-induced lung function loss. Cancer Res 65(15):6509-6511

15. Vedam SS, Keall PJ, Kini VR, Mostafavi H, Shukla HP, Mohan R (2003) Acquiring a four-dimensional computed tomography dataset using an external respiratory signal. Phys Med Biol 48(1):45-62

16. Hart JP, McCurdy MR, Ezhil M et al (2008) Radiation pneumonitis: correlation of toxicity with pulmonary metabolic radiation response. Int J Radiat Oncol Biol Phys 71(4):967-971

17. Guerrero T, Johnson V, Hart J et al (2007) Radiation pneumonitis: local dose versus $\left[{ }^{18} \mathrm{~F}\right]$-fluorodeoxyglucose uptake response in irradiated lung. Int J Radiat Oncol Biol Phys 68(4):1030-1035

18. Ajani JA, Komaki R, Putnam JB et al (2001) A three-step strategy of induction chemotherapy then chemoradiation followed by surgery in patients with potentially resectable carcinoma of the esophagus or gastroesophageal junction. Cancer 92(2):279-286

19. Pan T, Lee TY, Rietzel E, Chen GT (2004) 4D-CT imaging of a volume influenced by respiratory motion on multi-slice CT. Med Phys 31(2):333-340

20. Pan T, Mawlawi O, Luo D et al (2006) Attenuation correction of PET cardiac data with low-dose average CT in PET/CT. Med Phys 33(10):3931-3938

21. Ahnesjo A (1989) Collapsed cone convolution of radiant energy for photon dose calculation in heterogeneous media. Med Phys 16 (4):577-592

22. Theuws JCM, Kwa SLS, Wagenaara AC et al (1998) Dose-effect relations for early local pulmonary injury after irradiation for malignant lymphoma and breast cancer. Radiother Oncol 48 (1):33-43 